OPEN ACCESS

Edited by:

Michail Nomikos,

Qatar University, Qatar

Reviewed by:

Debora Cohen,

Consejo Nacional de Investigaciones Cientificas y Técnicas (CONICET),

Argentina

Karl Swann,

Cardiff University, United Kingdom

*Correspondence:

Carmen J. Williams williamsc5@niehs.nih.gov

Specialty section:

This article was submitted to Molecular and Cellular Reproduction, a section of the journal Frontiers in Cell and Developmental

Biology

Received: 20 August 2021 Accepted: 21 October 2021

Published: 04 November 2021

Citation:

Savy V, Stein P, Shi M and Williams $C J$ (2021) Superovulation Does Not Alter

Calcium Oscillations

Following Fertilization.

Front. Cell Dev. Biol. 9:762057.

doi: 10.3389/fcell.2021.762057

\section{Superovulation Does Not Alter Calcium Oscillations Following Fertilization}

\author{
Virginia Savy ${ }^{1}$, Paula Stein ${ }^{1}$, Min Shi ${ }^{2}$ and Carmen J. Williams ${ }^{1 *}$ \\ ${ }^{1}$ Reproductive and Developmental Biology Laboratory, Durham, NC, United States, ${ }^{2}$ Biostatistics and Computational Biology \\ Branch, National Institute of Environmental Health Sciences, National Institutes of Health, Durham, NC, United States
}

Superovulation is a common approach to maximize the number of eggs available for either clinical assisted reproductive technologies or experimental animal studies. This procedure provides supraphysiological amounts of gonadotropins to promote continued growth and maturation of ovarian follicles that otherwise would undergo atresia. There is evidence in mice, cows, sheep, and humans that superovulation has a detrimental impact on the quality of the resulting ovulated eggs or embryos. Here we tested the hypothesis that eggs derived from superovulation have a reduced capacity to support calcium oscillations, which are a critical factor in the success of embryo development. Eggs were obtained from mice that were either naturally cycling or underwent a standard superovulation protocol. The eggs were either parthenogenetically activated using strontium or fertilized in vitro while undergoing monitoring of calcium oscillatory patterns. Following parthenogenetic activation, superovulated eggs had a slightly delayed onset and longer duration of the first calcium transient, but no differences in oscillation persistence, frequency, or total calcium signal. However, in vitro fertilized superovulated eggs had no differences in any of these measures of calcium oscillatory behavior relative to spontaneously ovulated eggs. These findings indicate that although subtle differences in calcium signaling can be detected following parthenogenetic activation, superovulation does not disrupt physiological calcium signaling at fertilization, supporting the use of this method for both clinical and experimental purposes.

Keywords: superovulation, oocyte, mouse, calcium oscillations, egg activation

\section{INTRODUCTION}

Fertilization represents the union of two terminally differentiated gametes to form a single embryo capable of developing into a unique individual. Gamete fusion is only the beginning of the process of embryo development, but it sets in motion a series of events collectively termed "egg activation" that turn the unified gametes into a totipotent embryo capable of becoming a healthy individual. The key event of egg activation that triggers development in all species is a rise in the cytoplasmic calcium level (Kashir et al., 2013). What is unusual about mammalian eggs is that following the initial calcium rise, they go on to have a series of oscillations in cytoplasmic calcium levels that persist for several hours after fertilization. These calcium oscillations drive downstream events of egg activation including exocytosis of cortical granules, activation of calcium/calmodulin-dependent protein kinase II-gamma, resumption of the cell cycle, and pronucleus formation, all of which are essential for initiating proper embryo development (Stein et al., 2020). Persistent calcium oscillations can be 
triggered in the absence of sperm using the divalent cation strontium, resulting in parthenogenetic egg activation mediated by the transient receptor potential channel TRPV3 (Fraser, 1987; Carvacho et al., 2013). In the mouse, an inappropriate pattern of calcium oscillations following fertilization is associated with reductions in implantation efficiency, reduced development to term, and abnormalities in offspring growth (Ozil and Huneau, 2001; Ducibella et al., 2002; Ozil et al., 2005, 2006).

The pattern of calcium oscillations at fertilization is modulated by the amount of PLCל released by the sperm but also by many factors intrinsic to the fertilized egg (Stein et al., 2020). Calcium oscillations depend on the amount of calcium in endoplasmic reticulum (ER) stores as well as egg factors that regulate how quickly calcium is released from the ER, cleared from the cytoplasm, and then pumped back into the ER such that sufficient calcium stores are available for the next calcium release event. Calcium homeostasis is regulated by the activities of calcium pumps and ion channels (Berridge et al., 2003). Sarco-endoplasmic reticulum calcium-ATPases pump calcium back into the ER, and plasma membrane calciumATPase pumps clear calcium from the cytoplasm by extruding it across the plasma membrane. These pumps depend on mitochondrial production of ATP. Calcium influx channels support calcium entry into the cytoplasm down a concentration gradient from the extracellular milieu; calcium influx is necessary for complete refilling of ER stores and continuation of calcium oscillations (Igusa and Miyazaki, 1983; Kline and Kline, 1992). Together, these activities allow the egg to tolerate the release of large amounts of calcium from the ER and to refill ER stores once the calcium release event has been completed.

All the molecular components needed to support calcium homeostasis, egg activation, and preimplantation embryo development, such as stored proteins, nucleic acids, and energy substrates, are generated during the oocyte growth phase. In a normal estrous cycle, ovarian follicles are recruited into the growing pool independent of gonadotropin hormones until the secondary follicle stage, when the oocyte is surrounded by several layers of granulosa cells (Strauss and Williams, 2017). Follicle stimulating hormone (FSH), secreted from the pituitary, initiates further follicle development to the antral stage, when fluid begins to collect between the granulosa cells. FSH and luteinizing hormone $(\mathrm{LH})$ together promote continued survival and development of antral follicles toward the preovulatory stage (Chun et al., 1996; Sullivan et al., 1999). When FSH levels decline in response to estradiol-mediated negative feedback on the pituitary, antral follicles instead will undergo atresia. A select few "dominant" follicles continue to grow despite declining FSH levels. These follicles are either more sensitive to the available FSH or have developed sufficient responsiveness to $\mathrm{LH}$ to continue their developmental trajectory (Sullivan et al., 1999). As a result, a species-specific number of preovulatory follicles forms during each estrous cycle.

Successful superovulation was first reported almost 100 years ago, when whole pituitary tissue from donor animals was injected intramuscularly into rats and mice and resulted in either ovaries containing very large numbers of normal-appearing follicles or oviducts containing large numbers of ovulated eggs (Smith and Engle, 1927). Although the exact procedure has been modified extensively since that time, superovulation is commonly utilized to maximize the number of ovulated eggs available for either experimental purposes or for clinical use within assisted reproduction cycles. Superovulation works by providing high amounts of exogenous gonadotropin hormone so that nondominant late secondary and early antral follicles, which are sensitive to gonadotropin stimulation and would otherwise be destined to undergo atresia, are instead maintained in the growing follicle pool and form preovulatory follicles. The preovulatory follicles can be allowed to ovulate spontaneously or induced to ovulate by administration of chorionic gonadotropin, which binds to the $\mathrm{LH}$ receptor and begins the ovulatory signaling cascade.

There is evidence in mice, cows, sheep, and humans that superovulation has a detrimental impact on the quality of the resulting ovulated eggs or embryos (Moor et al., 1985; Hyttel et al., 1986; Yun et al., 1989; Assey et al., 1994; Blondin et al., 1996; Van der Auwera, 2001; Baart et al., 2007; Fortier et al., 2008; Market-Velker et al., 2010; Lee et al., 2017). These findings could be explained by many factors, including accelerated oocyte growth, rescue of abnormal oocytes from atresia, and recruitment for ovulation of non-mature oocytes, all of which could lead to eggs that lack the maternally derived stores needed for developmental competence. Because calcium oscillatory patterns depend heavily on maternal components that regulate calcium homeostasis, we hypothesized that superovulation results in eggs that have abnormal patterns of calcium oscillations following parthenogenetic activation or fertilization. Small differences in calcium oscillatory behavior between spontaneously ovulated and superovulated eggs were observed following parthenogenetic activation, but there were no differences following in vitro fertilization (IVF). These findings indicate that calcium responses to sperm-induced egg activation are sufficiently robust to overcome subtle deficits in egg competence associated with superovulation.

\section{METHODS}

\section{Animals and Superovulation}

C57BL/6J females (3-6 weeks old), C57BL/6J vasectomized males (2-8 months old), and B6SJLF1/J males (4-6 months old) were obtained from The Jackson Laboratory (Bar Harbor, ME).

Metaphase II-arrested (MII) eggs were collected from the oviducts of naturally cycling, 6-week-old females following overnight breeding to vasectomized males; vaginal plugs were checked to assure ovulation had occurred. For superovulation, 3week-old females were primed by intraperitoneal injection of 5 IU of equine chorionic gonadotropin (Lee Biosolutions, Maryland Heights, MO) followed $46-48 \mathrm{~h}$ later by 5 IU human chorionic gonadotropin (Sigma Aldrich, St. Louis, MO). The superovulated females were also bred overnight to vasectomized males for consistency across both treatment groups; only vaginal plugpositive females were used. 
All mice were sacrificed by $\mathrm{CO}_{2}$ asphyxiation and cervical dislocation. All procedures involving mice were conducted in accordance with National Institute of Environmental Health Sciences guidelines under approved animal care and use protocols.

\section{Parthenogenetic Activation, in Vitro Fertilization and Calcium Imaging}

Eggs were collected at 9:00 am on the day the vaginal plug was detected, which corresponded to $14 \mathrm{~h}$ after human chorionic gonadotropin injection of superovulated females. Minimal Essential Medium with Hepes (Thermo Fisher, Waltham, MA) containing $0.1 \%$ PVA and $0.1 \%$ hyaluronidase (Sigma, St. Louis, MO) was used for egg collection and cumulus cell removal. In each biological replicate, eggs from 3-5 mice, either superovulated or naturally cycling, were pooled and treated with acidic Tyrode's solution ( $\mathrm{pH}$ 1.6) to remove the zona pellucida. The zona-free eggs were allowed to recover in KSOM medium (Millipore Sigma, Burlington, MA) for $30 \mathrm{~min}$. The eggs were then loaded with the calcium indicator Fura-2 AM ( $5 \mu \mathrm{M}$; Thermo Fisher) for $30 \mathrm{~min}$ in $\mathrm{KSOM}$ containing $0.02 \%$ pluronic F-127 (Thermo Fisher) and then washed in fresh KSOM medium.

To monitor changes in cytosolic calcium, eggs obtained from superovulated or naturally cycling mice were adhered side by side to glass-bottom dishes (MatTek, Ashland, MA). This procedure ensured that the eggs in the two groups were cultured under exactly the same conditions. For parthenogenetic activation, eggs were adhered in $1.8 \mathrm{ml}$ of $\mathrm{Ca}^{2+} / \mathrm{Mg}^{2+}$-free $\mathrm{CZB}$ medium (Gambini et al., 2020) without polyvinyl alcohol and, after calcium imaging was initiated, strontium $(200 \mu \mathrm{l}$ of $100 \mathrm{mM}$ $\mathrm{SrCl}_{2}$ in $\mathrm{Ca}^{2+} / \mathrm{Mg}^{2+}$-free $\mathrm{CZB}$ ) was added to achieve a final concentration of $10 \mathrm{mM}$. For IVF, eggs were adhered to CellTak-treated (Thermo Fisher) dishes in $150 \mu$ l of BSA-free KSOM (Millipore Sigma). Forty-five $\mu \mathrm{l}$ of human tubal fluid medium (HTF; Millipore Sigma) containing $4 \mathrm{mg} / \mathrm{ml}$ BSA (HTF-BSA) were added to the drop to achieve a final concentration, once sperm were added, of $1 \mathrm{mg} / \mathrm{ml} \mathrm{BSA}$, and then the drop was covered with mineral oil.

Motile sperm were isolated from the epididymides of adult B6SJLF1/J males in HTF-BSA, via the swim-up method. Briefly, the epididymis and vas deferens were dissected and placed in $500 \mu \mathrm{l}$ HTF-BSA covered with mineral oil. Under a dissection microscope, the tissue was cut several times and cultured for $15 \mathrm{~min}$ at $37^{\circ} \mathrm{C}$ to allow the motile sperm to swim out. The tissue was removed and $150 \mu \mathrm{l}$ of swim-out sperm were carefully placed at the bottom of a clean tube containing $850 \mu \mathrm{l}$ of HTF-BSA. The sperm were cultured for $60 \mathrm{~min}$ at $37^{\circ} \mathrm{C}$ to allow the motile sperm to swim up and capacitate. Sperm from the top layer of the culture medium were carefully transferred to a pre-warmed tube and the final sperm concentration was determined using a hemocytometer. Sperm were diluted in HTF-BSA to $10^{6}$ sperm $/ \mathrm{ml}$, then $5 \mu \mathrm{l}$ were added to the IVF dish $(195 \mu \mathrm{l}$ volume) to achieve a final concentration of $25,000 \mathrm{sperm} / \mathrm{ml}$.

Cytosolic calcium was measured by recording the intensity of fluorescence induced by 340 and $380 \mathrm{~nm}$ excitation and calculating the F340/F380 ratio. Ratio images were recorded every $7.5 \mathrm{~s}$ using a Hamamatsu ORCA-Flash4.0 LT + digital camera (Hamamatsu, Bridgewater, NJ) attached to a Nikon Ti inverted microscope with a Nikon S Fluor 20x/0.75 NA objective (Nikon Instruments, Melville, NY) and a Lambda 10-B Optical Filter Changer (Sutter Instruments, Novato, CA). Nikon NISElements software was used for data acquisition and visualization. All experiments were performed at $37^{\circ} \mathrm{C}$ in a humidified atmosphere of $5 \% \quad \mathrm{CO}_{2}$, in an Okolab stage microenvironmental chamber enclosed in a microscope cage incubator (Okolab, Ambridge, PA).

For parthenogenetic activation, a total of four biological replicates and eight technical replicates were analyzed, including 78 eggs from superovulated females and 75 eggs from naturally cycling females. For IVF, a total of four biological replicates and eight technical replicates were analyzed, including 92 eggs from superovulated females and 68 eggs from naturally cycling females. Eggs of each group were either activated with strontium or fertilized and imaged at the same time, in the same medium drop, for each experimental replicate.

\section{Data Analysis and Statistical Tests}

Significance of differences between the number of eggs obtained from naturally cycling and superovulated females was tested using the Mann-Whitney $U$-test, as the data were not normally distributed. The analysis was performed at significance level of 0.05 and CI of 95\%, using Prism version 9.0.2.

A custom $\mathrm{R}$ function was developed for automated analysis of calcium imaging data and is available at https://www.niehs.nih. gov/research/atniehs/labs/assets/docs/q_z/savy_rscript.zip. The R-Script was used to determine the time to the first calcium transient, length of the first oscillation, area under the curve (AUC), number of oscillations for 60 and $120 \mathrm{~min}$ from the starting point of the first transient, number of oscillations per $10 \mathrm{~min}$ and persistence of oscillations for 110-120 min. Statistical tests were performed using GraphPad Prism. Because the data were not normally distributed, the time to the first transient, length of the first transient, AUC, and oscillation frequency were analyzed using a Mann-Whitney $U$-test. Data regarding oscillation persistence were analyzed using the Log-rank (Mantel-Cox) test.

\section{RESULTS}

We used the inbred mouse line, C57BL/6J, to test whether there were differences in calcium oscillatory patterns between eggs from naturally cycling (NC) and superovulated (SOV) mice. This choice was driven in large part because knockout facilities utilize this strain for the generation of the majority of their animal models. Furthermore, many transgenic and knockout lines are uniquely available on this background strain. As anticipated, far more eggs were obtained from SOV mice (median $=60.5$ eggs) than from NC mice (median $=8$ eggs; Mann-Whitney $\mathrm{U}=41$, $\mathrm{n}_{\mathrm{NC}}=20 \mathrm{n}_{\mathrm{SOV}}=22, p<0.0001$; two-tailed).

We first tested SOV and NC eggs for differences in calcium oscillatory patterns following strontium-induced parthenogenetic 

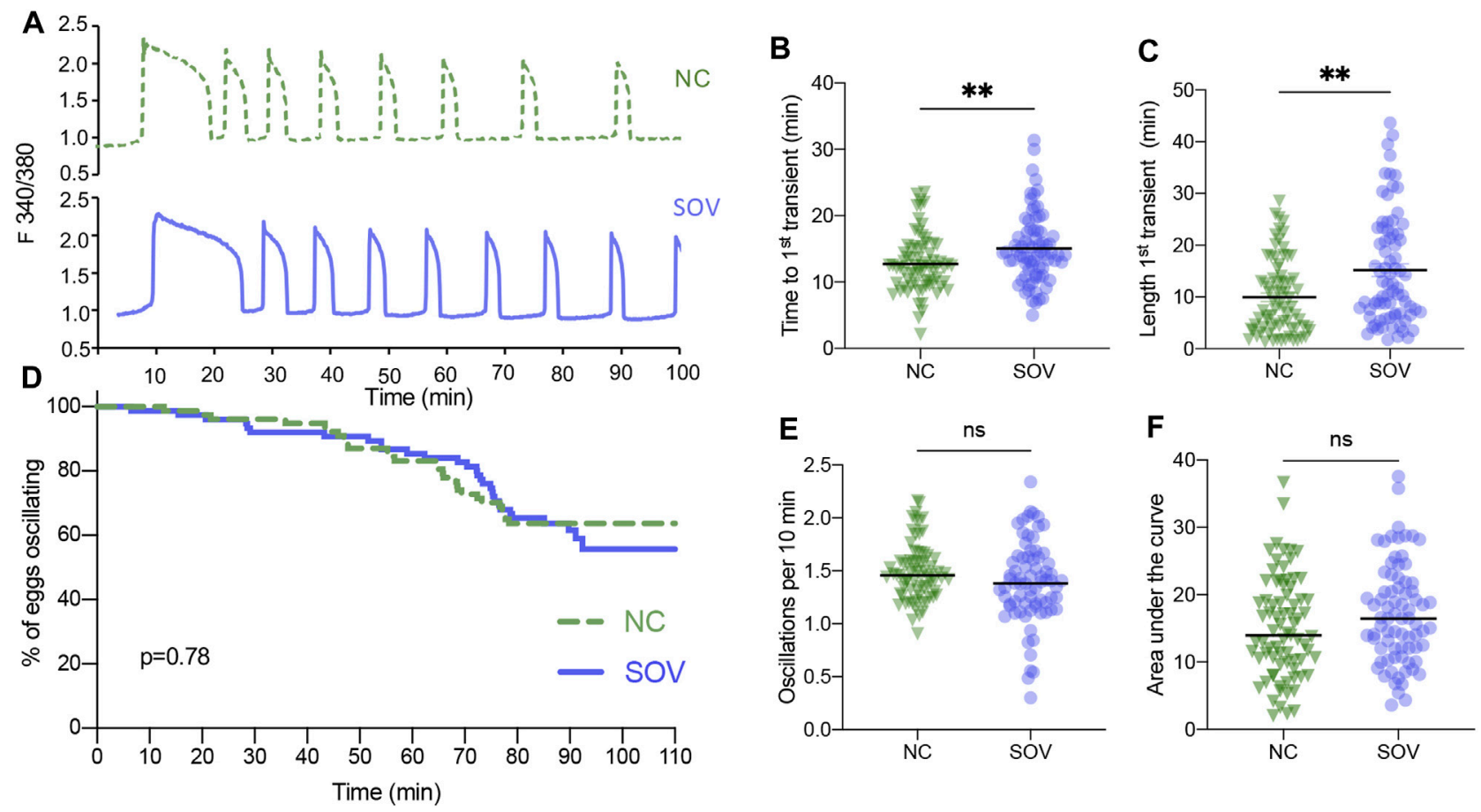

FIGURE 1 | Calcium oscillatory patterns following strontium-induced parthenogenetic activation of MII eggs from naturally cycling (NC) and superovulated (SOV) mice. (A) Representative calcium traces. (B) Time to the first transient. (C) Length of the first calcium transient. (D) Percentage of eggs that continued to display calcium oscillations during 110 min. p-value indicated on graph. (E) Oscillation frequency averaged over the first 60 min of oscillations. (F) Area under the curve of calcium signal during the first 60 min following activation. Graphs in (B,C,E,F) show median and all individual data points. ${ }^{\star *} p<0.01$; ns, no significant difference.

activation, which prevents any potential variability that could be introduced by differences in individual sperm during IVF. SOV eggs took slightly longer to begin oscillating and the first transient was about 50\% longer (Figures 1A-C), indicating that there were differences in strontium sensitivity and in cytoplasmic calcium handling in the presence of strontium. A detailed analysis of additional parameters including the percentage of eggs that continued to display calcium oscillations (oscillation persistence), oscillation frequency, and the area under the curve of calcium signal revealed no significant differences between the two groups (Figures 1D-F).

A similar analysis was performed of the calcium oscillatory responses of SOV and NC eggs to sperm-induced egg activation. There were no obvious differences in the patterns of calcium oscillations, the time until the first transient, or the duration of the first transient (Figures 2A-C). Likewise, there were no significant differences in calcium oscillation persistence, frequency, or total calcium signal (Figures 2D-F). These findings suggest that superovulation does not impact fertilization-induced calcium oscillatory patterns to any measurable degree.

\section{DISCUSSION}

Here we tested in a highly controlled fashion whether superovulation causes abnormalities in calcium signaling following strontium-induced parthenogenetic activation or IVF in the mouse. We found that strontium-induced oscillations were slightly different in superovulated eggs, with a small delay in initiation and longer total duration of the first transient. Because TRPV3 mediates strontium-induced egg activation, differences in the amount of TRPV3 on the egg surface could explain the altered time to the first transient (Carvacho et al., 2013). In addition, differences in the ability of the egg to clear large amounts of calcium from the cytoplasmic compartment in the presence of strontium could explain the prolonged first transient. Our findings are similar to previous observations that eggs from aged mice differ in some of their calcium oscillatory responses to strontium relative to those in eggs from young mice, whereas these differences are not observed following more physiological egg activation methods (microinjection of either sperm heads or

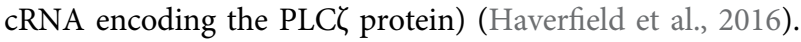

Despite our prediction to the contrary, the data clearly showed that in a commonly utilized inbred strain, C57BL/6J, superovulation according to a standard protocol does not alter the calcium oscillatory patterns at fertilization. It is possible that alternate superovulation protocols or repetitive superovulation could produce a different result. Superovulation maximizes the numbers of ovulated eggs that can be obtained for study from each mouse, reducing the numbers of animals that must be euthanized for experimental purposes by about 10-fold based on our data. Therefore, this technique is routinely performed to obtain oocytes and early embryos in laboratories and animal facilities. Our findings demonstrating normal 

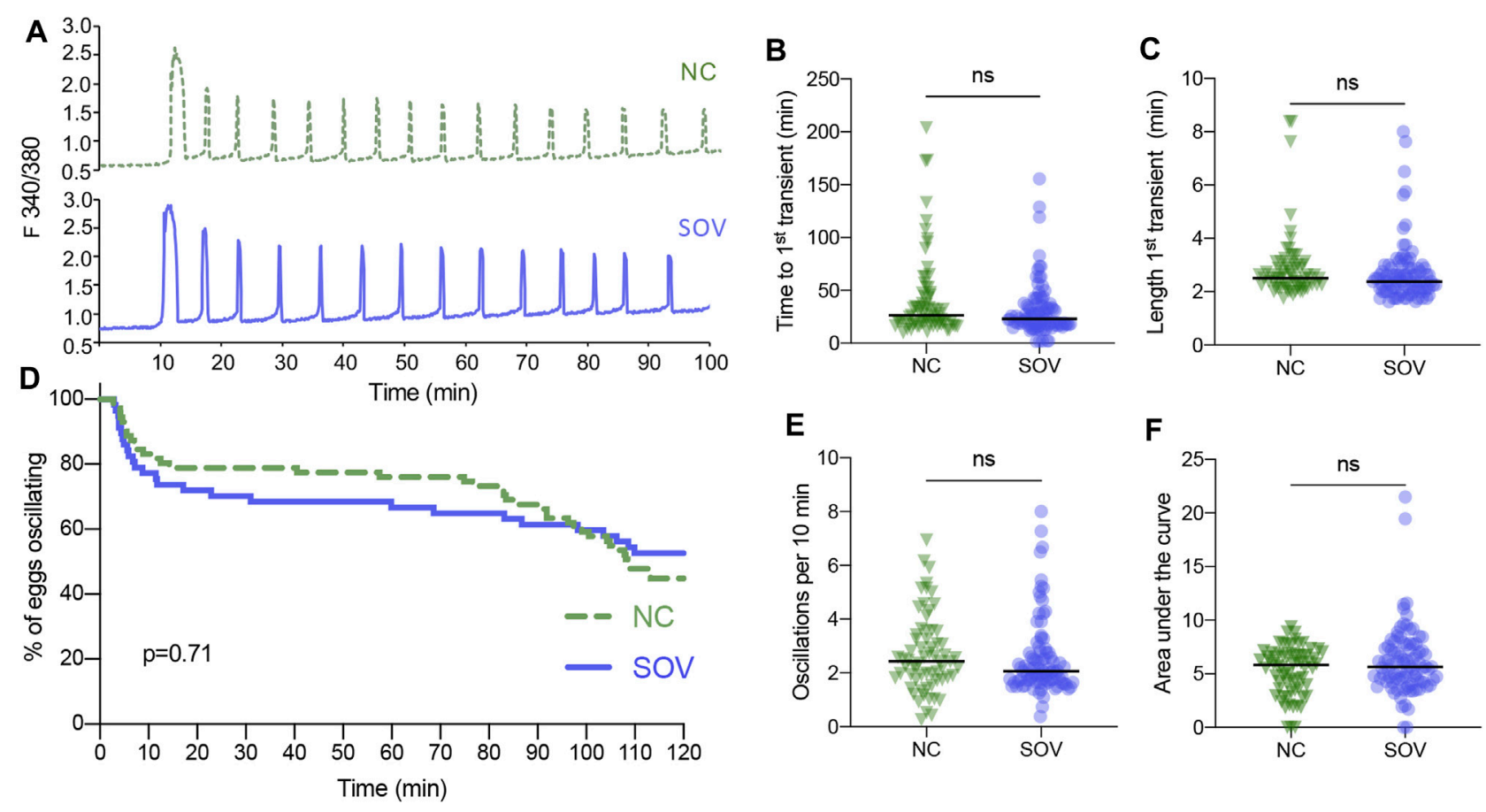

FIGURE 2 | Calcium oscillatory patterns following fertilization of Mll eggs from naturally cycling (NC) and superovulated (SOV) mice. (A) Representative calcium traces. (B) Time to the first transient. (C) Length of the first calcium transient. (D) Percentage of eggs that continued to display calcium oscillations during 120 min. p-value indicated on graph. (E) Oscillation frequency averaged over the first 60 min of oscillations. (F) Area under the curve of calcium signal during the first 60 min following fertilization. Graphs in (B,C,E,F) show median and all individual data points. ns, no significant difference.

fertilization-induced calcium oscillatory patterns despite superovulation is reassuring regarding interpretation of experiments examining factors that influence calcium signals given that these types of experiments are almost always performed with eggs obtained by superovulation. Our data support the use of superovulation in future studies of calcium signaling at fertilization, but suggest caution in the interpretation of results obtained in experiments utilizing chemical methods to activate superovulated eggs.

Despite being a widespread practice, superovulation is associated with abnormalities in several phenotypic features in oocytes and ovulated eggs. For example, an increased incidence of meiosis arrest prior to metaphase II, asynchrony between nuclear and cytoplasmic maturation, abnormalities in subcellular structure, and abnormal patterns of protein synthesis have all been observed in various mammalian species following superovulation (Moor et al., 1985; Callesen et al., 1986; Hyttel et al., 1986; Yun et al., 1989; Assey et al., 1994). Given that the hormone injections only rescue late secondary and antral follicles, these differences are clearly a consequence of inadequate preparation for oocyte maturation during later stages of follicle development. Although the oocyte is transcriptionally inactive after reaching the antral stage, translation continues to occur, creating additional maternal components to support maturation, and embryo development. In addition, mRNAs and long noncoding RNAs are transferred to the oocyte from the surrounding cumulus cells, at least some of which are translated and may contribute to oocyte competence to undergo maturation (Macaulay et al., 2014, 2016). Superovulation could accelerate late follicle development enough that there is insufficient time for the accumulation of specific maternal components that could explain the altered sensitivity to strontium in SOV and NC eggs. However, the absence of differences in fertilization-induced calcium dynamics suggests that adequate amounts of the maternal components required for physiological calcium homeostasis are already present prior to superovulation or, alternatively, that these components are not affected in a detrimental fashion by the superovulation protocol.

Unlike oocyte maturation defects, which occur prior to fertilization, some superovulation-associated abnormalities are observed following fertilization. Reduced competence to develop to or beyond the 16-cell stage, both in vivo and in vitro, abnormalities in acquisition and maintenance of methylation marks on imprinted genes and fetal growth retardation all are associated with superovulation protocols in cows and/or mice (Blondin et al., 1996; Van der Auwera, 2001; Fortier et al., 2008; Market-Velker et al., 2010). Differences in embryo development likely result from a lack of accumulation of other maternal components in the final period of follicle growth to the periovulatory stage that are not related to the calcium signaling toolkit. Future studies will be needed to determine the identity of such components or to develop superovulation protocols that do not disrupt preovulatory follicle development. 


\section{DATA AVAILABILITY STATEMENT}

The raw data supporting the conclusion of this article will be made available by the authors, without undue reservation.

\section{ETHICS STATEMENT}

The animal study was reviewed and approved by the Animal Care and Use Committee, National Institute of Environmental Health Sciences.

\section{AUTHOR CONTRIBUTIONS}

VS, PS, and CW designed the study. VS and PS conducted the experiments. MS wrote software for calcium signal analyses. VS

\section{REFERENCES}

Assey, R. J., Hyttel, P., Roche, J. F., and Boland, M. (1994). Oocyte Structure and Follicular Steroid Concentrations in Superovulated versus Unstimulated Heifers. Mol. Reprod. Dev. 39, 8-16. doi:10.1002/mrd.1080390103

Baart, E. B., Martini, E., Eijkemans, M. J., Van Opstal, D., Beckers, N. G. M., Verhoeff, A., et al. (2007). Milder Ovarian Stimulation for In-Vitro Fertilization Reduces Aneuploidy in the Human Preimplantation Embryo: a Randomized Controlled Trial. Hum. Reprod. 22, 980-988. doi:10.1093/humrep/del484

Berridge, M. J., Bootman, M. D., and Roderick, H. L. (2003). Calcium Signalling: Dynamics, Homeostasis and Remodelling. Nat. Rev. Mol. Cell Biol. 4, 517-529. doi:10.1038/nrm1155

Blondin, P., Coenen, K., Guilbault, L. A., and Sirard, M.-A. (1996). Superovulation Can Reduce the Developmental Competence of Bovine Embryos. Theriogenology 46, 1191-1203. doi:10.1016/S0093-691X(96) 00290-7

Callesen, H., Greve, T., and Hyttel, P. (1986). Preovulatory Endocrinology and Oocyte Maturation in Superovulated Cattle. Theriogenology 25, 71-86. doi:10.1016/0093-691X(86)90184-6

Carvacho, I., Lee, H. C., Fissore, R. A., and Clapham, D. E. (2013). TRPV3 Channels Mediate Strontium-Induced Mouse-Egg Activation. Cell Rep. 5, 1375-1386. doi:10.1016/j.celrep.2013.11.007

Chun, S. Y., Eisenhauer, K. M., Minami, S., Billig, H., Perlas, E., and Hsueh, A. J. (1996). Hormonal Regulation of Apoptosis in Early Antral Follicles: FollicleStimulating Hormone as a Major Survival Factor. Endocrinology 137, 1447-1456. doi:10.1210/endo.137.4.8625923

Ducibella, T., Huneau, D., Angelichio, E., Xu, Z., Schultz, R. M., Kopf, G. S., et al. (2002). Egg-to-Embryo Transition Is Driven by Differential Responses to Ca2+ Oscillation Number. Developmental Biol. 250, 280-291. doi:10.1016/S00121606(02)90788-810.1006/dbio.2002.0788

Fortier, A. L., Lopes, F. L., Darricarrère, N., Martel, J., and Trasler, J. M. (2008). Superovulation Alters the Expression of Imprinted Genes in the Midgestation Mouse Placenta. Hum. Mol. Genet. 17, 1653-1665. doi:10.1093/hmg/ddn055

Fraser, L. R. (1987). Strontium Supports Capacitation and the Acrosome Reaction in Mouse Sperm and Rapidly Activates Mouse Eggs. Gamete Res. 18, 363-374. doi:10.1002/mrd.1120180410

Gambini, A., Stein, P., Savy, V., Grow, E. J., Papas, B. N., Zhang, Y., et al. (2020). Developmentally Programmed Tankyrase Activity Upregulates $\beta$-Catenin and Licenses Progression of Embryonic Genome Activation. Developmental Cell 53, 545-560. doi:10.1016/j.devcel.2020.04.018

Haverfield, J., Nakagawa, S., Love, D., Tsichlaki, E., Nomikos, M., Lai, F. A., et al. (2016). Ca2+ Dynamics in Oocytes from Naturally-Aged Mice. Sci. Rep. 6, 19357. doi:10.1038/srep19357

Hyttel, P., Callesen, H., and Greve, T. (1986). Ultrastructural Features of Preovulatory Oocyte Maturation in Superovulated Cattle. Reproduction 76, 645-656. doi:10.1530/jrf.0.0760645 analyzed the data. VS and CW wrote the first draft of the article. VS, PS, MS, and CW edited the article.

\section{FUNDING}

This work was supported by the Intramural Research Program of the National Institutes of Health, National Institute of Environmental Health Sciences, 1ZIAES102985.

\section{ACKNOWLEDGMENTS}

We thank Briggs Hagler and Greg Scott (NIEHS) for assistance with animal handling and Safia Malki and Karina Rodriguez (NIEHS) for critical review of the article.

Igusa, Y., and Miyazaki, S. (1983). Effects of Altered Extracellular and Intracellular Calcium Concentration on Hyperpolarizing Responses of the Hamster Egg. J. Physiol. 340, 611-632. doi:10.1113/jphysiol.1983.sp014783

Kashir, J., Deguchi, R., Jones, C., Coward, K., and Stricker, S. A. (2013). Comparative Biology of Sperm Factors and Fertilization-Induced Calcium Signals across the Animal Kingdom. Mol. Reprod. Dev. 80, 787-815. doi:10.1002/mrd.22222

Kline, D., and Kline, J. T. (1992). Thapsigargin Activates a Calcium Influx Pathway in the Unfertilized Mouse Egg and Suppresses Repetitive Calcium Transients in the Fertilized Egg. J. Biol. Chem. 267, 17624-17630. doi:10.1016/s0021-9258(19)37088-7

Lee, M., Ahn, J. I., Lee, A. R., Ko, D. W., Yang, W. S., Lee, G., et al. (2017). Adverse Effect of Superovulation Treatment on Maturation, Function and Ultrastructural Integrity of Murine Oocytes. Mol. Cell 40, 558-566. doi:10.14348/molcells.2017.0058

Macaulay, A. D., Gilbert, I., Caballero, J., Barreto, R., Fournier, E., Tossou, P., et al. (2014). The Gametic Synapse: RNA Transfer to the Bovine Oocytel. Biol. Reprod. 91, 1-12. doi:10.1095/biolreprod.114.119867

Macaulay, A. D., Gilbert, I., Scantland, S., Fournier, E., Ashkar, F., Bastien, A., et al. (2016). Cumulus Cell Transcripts Transit to the Bovine Oocyte in Preparation for Maturation1. Biol. Reprod. 94, 1-11. doi:10.1095/biolreprod.114.127571

Market-Velker, B. A., Zhang, L., Magri, L. S., Bonvissuto, A. C., and Mann, M. R. W. (2010). Dual Effects of Superovulation: Loss of Maternal and Paternal Imprinted Methylation in a Dose-dependent Manner. Hum. Mol. Genet. 19, 36-51. doi:10.1093/hmg/ddp465

Moor, R. M., Osborn, J. C., and Crosby, I. M. (1985). Gonadotrophin-induced Abnormalities in Sheep Oocytes after Superovulation. Reproduction 74, 167-172. doi:10.1530/jrf.0.0740167

Ozil, J.-P., Banrezes, B., Tóth, S., Pan, H., and Schultz, R. M. (2006). Ca2+ Oscillatory Pattern in Fertilized Mouse Eggs Affects Gene Expression and Development to Term. Developmental Biol. 300, 534-544. doi:10.1016/j.ydbio.2006.08.041

Ozil, J.-P., Markoulaki, S., Toth, S., Matson, S., Banrezes, B., Knott, J. G., et al. (2005). Egg Activation Events Are Regulated by the Duration of a Sustained [Ca2+]cyt Signal in the Mouse. Developmental Biol. 282, 39-54. doi:10.1016/j.ydbio.2005.02.035

Ozil, J. P., and Huneau, D. (2001). Activation of Rabbit Oocytes: the Impact of the Ca2+ Signal Regime on Development. Development 128, 917-928. doi:10.1242/ dev.128.6.917

Smith, P. E., and Engle, E. T. (1927). Experimental Evidence Regarding the Rôle of the Anterior Pituitary in the Development and Regulation of the Genital System. Am. J. Anat. 40, 159-217. doi:10.1002/aja.1000400202

Stein, P., Savy, V., Williams, A. M., and Williams, C. J. (2020). Modulators of Calcium Signalling at Fertilization. Open Biol. 10, 200118. doi:10.1098/ rsob.200118rsob200118

Strauss, J. F., III, and Williams, C. J. (2017). “Ovarian Life Cycle,” in Yen \& Jaffe's Reproductive Endocrinology: Physiology, Pathophysiology, and Clinical Management. Editors R. L. Barbieri, J. F. StraussIII, and A. R. Gargiulo (Philadelphia, PA: Elsevier), 167-205.

Sullivan, M. W., Stewart-Akers, A., Krasnow, J. S., Berga, S. L., and Zeleznik, A. J. (1999). Ovarian Responses in Women to Recombinant Follicle-Stimulating Hormone and Luteinizing Hormone (LH): A Role for $\mathrm{LH}$ in the Final Stages of Follicular 
Maturation1. J. Clin. Endocrinol. Metab. 84, 228-232. doi:10.1210/ jc.84.1.22810.1210/jcem.84.1.5389

Van der Auwera, I. (2001). Superovulation of Female Mice Delays Embryonic and Fetal Development. Hum. Reprod. 16, 1237-1243. doi:10.1093/humrep/16.6.1237

Yun, Y. W., Yu, F. H., Yuen, B. H., and Moon, Y. S. (1989). Effects of a Superovulatory Dose of Pregnant Mare Serum Gonadotropin on Follicular Steroid Contents and Oocyte Maturation in Rats. Gamete Res. 23, 289-298. doi:10.1002/mrd.1120230306

Conflict of Interest: The authors declare that the research was conducted in the absence of any commercial or financial relationships that could be construed as a potential conflict of interest.
Publisher's Note: All claims expressed in this article are solely those of the authors and do not necessarily represent those of their affiliated organizations, or those of the publisher, the editors and the reviewers. Any product that may be evaluated in this article, or claim that may be made by its manufacturer, is not guaranteed or endorsed by the publisher.

Copyright (c) 2021 Savy, Stein, Shi and Williams. This is an open-access article distributed under the terms of the Creative Commons Attribution License (CC BY). The use, distribution or reproduction in other forums is permitted, provided the original author(s) and the copyright owner(s) are credited and that the original publication in this journal is cited, in accordance with accepted academic practice. No use, distribution or reproduction is permitted which does not comply with these terms. 